\title{
ROBUSTNESS OF DELTA HEDGING FOR PATH-DEPENDENT OPTIONS IN LOCAL VOLATILITY MODELS
}

\author{
ALEXANDER SCHIED,* TU Berlin \\ MITJA STADJE, ${ }^{* *}$ Princeton University
}

\begin{abstract}
We consider the performance of the delta hedging strategy obtained from a local volatility model when using as input the physical prices instead of the model price process. This hedging strategy is called robust if it yields a superhedge as soon as the local volatility model overestimates the market volatility. We show that robustness holds for a standard Black-Scholes model whenever we hedge a path-dependent derivative with a convex payoff function. In a genuine local volatility model the situation is shown to be less stable: robustness can break down for many relevant convex payoffs including averagestrike Asian options, lookback puts, floating-strike forward starts, and their aggregated cliquets. Furthermore, we prove that a sufficient condition for the robustness in every local volatility model is the directional convexity of the payoff function.
\end{abstract}

Keywords: Delta hedging; local volatility; robustness; directional convexity

2000 Mathematics Subject Classification: Primary 91B28

Secondary $60 \mathrm{H} 05 ; 60 \mathrm{H} 10$

\section{Introduction}

One of the key features of local volatility models is their completeness: in the model world every contingent claim admits a perfect hedge in terms of its delta hedging strategy. But what is the performance of this delta hedge if we use as input the physical prices quoted at the stock exchange instead of the model price process? Following El Karoui et al. [8], we will say that the delta hedging strategy is robust if this physical delta hedge is a superhedge for the claim as soon as the local volatility model overestimates the market volatility.

In the case of a European option, $h\left(S_{T}\right)$, it was shown in [8] that the convexity of $h$ is a sufficient condition for the robustness of the delta hedge in any reasonable local volatility model. This result is closely related to volatility comparison techniques as introduced by Hajek [15] and El Karoui et al. [8]. These techniques have since been generalized to multivariate price processes and processes with jumps; see [1], [7], [13], [17], [18], [19], and the references therein. See also Lyons [21] for an analytic, 'probability-free' result. This result relies on a setup for the physical price process, which is based on Föllmer's pathwise Itô formula [9], and thus, in this sense, is similar to ours. Volatility comparison techniques are also known to work in the context of American options [8] and can be translated to drift comparison via the Girsanov

Received 21 November 2006; revision received 20 September 2007.

* Postal address: Institut für Mathematik, TU Berlin, MA 7-4, Strasse des 17.Juni 136, 10623 Berlin, Germany.

Email address: schied@math.tu-berlin.de

** Postal address: Department of Operations Research and Financial Engineering, Princeton University, Engineering Quadrangle, Princeton, NJ 08544, USA. Email address: mstadje@ princeton.edu 
transform [22]. While many authors have used this technique to obtain ordering results for prices of contingent claims, our emphasis will rather be on hedging issues.

In this paper we study the robustness of the delta hedging strategy for general path-dependent derivatives of the form $h\left(S_{t_{1}}, \ldots, S_{t_{n}}\right)$. In Corollary 2.1 we show that the convexity of $h$ is a sufficient condition for robustness if the local volatility does not depend on the stock, i.e. if we are working with a Black-Scholes model. This positive result applies to a large number of standard path-dependent options including average-strike and average-price Asian options, lookback puts, floating-strike forward starts, and their aggregated cliquets. Perhaps this fact might help to explain the good hedging performance of the Black-Scholes model, which is sometimes reported by practitioners.

In a genuine local volatility model, however, the situation can be more complicated and less stable. In Theorem 2.1 we consider a reasonable family of local volatility models, in which robustness breaks down for many relevant path-dependent derivatives including average-strike Asian options, lookback puts, floating-strike forward starts, and their aggregated cliquets, even though they all correspond to convex payoff functions. The volatility functions for which our result is valid include a frequently observed pattern of empirical local volatilities in many equity markets.

In Theorem 2.2 we give a sufficient criterion on the payoff function $h$ under which the delta hedging strategy is robust for any reasonable local volatility model. This condition is the directional convexity of $h$, which is also analyzed by Bergenthum and Rüschendorf [1] in a multivariate though single-time setting. Directional convexity neither implies nor is implied by convexity in the usual sense. It applies, for instance, to the payoff function of an average-price Asian call.

In Section 2 we describe our setup and state our main results. Most proofs are deferred to Section 3.

\section{Statement of results}

A common approach to valuing exotic derivatives is to use a diffusion process $S=\left(S_{t}\right)_{t \geq 0}$ based on local volatility for modeling the risk-neutral evolution of the forward price of the underlying stock. The term 'local volatility' means that the instantaneous volatility at time $t$ is given as a function $\sigma\left(t, S_{t}\right)$ of $t$ and $S_{t}$ alone. That is, $S$ is a solution of the stochastic differential equation

$$
\mathrm{d} S_{t}=\sigma\left(t, S_{t}\right) S_{t} \mathrm{~d} W_{t}
$$

for a standard Brownian motion $W$. For simplicity, we will henceforth assume that the riskfree interest rate and all dividend payments are 0 , so that we need not distinguish between the underlying stock and its forward price. This is possible, without loss of generality, if the discount factor is deterministic.

In practice, the volatility function $\sigma(t, x)$ is often chosen in such a way that the local volatility model is calibrated to the market prices of liquid plain-vanilla options. Calibration can be achieved by combining the Dupire formula [6] (see also [14]) with an appropriate interpolation method. This approach guarantees that all European derivatives with payoff $h\left(S_{t}\right)$ are priced consistently with all plain-vanilla instruments.

Once the local volatility model is set up, it is typically used for the analysis of path-dependent exotic derivatives. In practice, the payoff of such a derivative is of the form

$$
H(S)=h\left(S_{t_{1}}, \ldots, S_{t_{n}}\right),
$$


where $0=t_{0}<t_{1}<t_{2}<\cdots<t_{n} \leq T$ and $h \geq 0$. In this paper we will mainly-but not exclusively - be interested in derivatives whose payoff function $h$ is convex. Standard examples include average-price Asian put and call options, with strike $K$,

$$
\left(K-\frac{1}{n} \sum_{i=1}^{n} S_{t_{i}}\right)^{+}, \quad\left(\frac{1}{n} \sum_{i=1}^{n} S_{t_{i}}-K\right)^{+},
$$

average-strike Asian options,

$$
\left(\frac{1}{n} \sum_{i=1}^{n} S_{t_{i}}-S_{T}\right)^{+}, \quad\left(S_{T}-\frac{1}{n} \sum_{i=1}^{n} S_{t_{i}}\right)^{+},
$$

lookback put options,

$$
\max _{i=1, \ldots, n} S_{t_{i}}
$$

floating-strike forward starting put and call options,

$$
\left(K S_{T_{0}}-S_{T}\right)^{+}, \quad\left(S_{T}-K S_{T_{0}}\right)^{+}, \quad \text { for } 0<T_{0}<T,
$$

and their aggregated cliquets,

$$
\sum_{i=1}^{n}\left(K S_{t_{i-1}}-S_{t_{i}}\right)^{+}, \quad \sum_{i=1}^{n}\left(S_{t_{i}}-K S_{t_{i-1}}\right)^{+} .
$$

In the sequel we will distinguish between the model $S_{t}$ of the stock price and the actual data quoted at the stock exchange. This physical price quoted at time $t$ will be denoted by $X_{t}$. We assume that such a price $X_{t}$ is available at any time $t \in[0, T]$. Since there is only a single quote of the stock at a given time and no control experiment is possible, it is natural to think of $X=\left(X_{t}\right)_{0 \leq t \leq T}$ as a fixed function on the time interval $[0, T]$ and not as a stochastic process involving additional randomization. In doing so, we follow the ideas of Föllmer [10], [11]. Thus, for a derivative with payoff function $h$, we denote its physical payoff by $h\left(X_{t_{1}}, \ldots, X_{t_{n}}\right)$, while it is modeled as the random variable $h\left(S_{t_{1}}, \ldots, S_{t_{n}}\right)$ in the local volatility model. It will sometimes be convenient to write $h\left(X_{t_{1}}, \ldots, X_{t_{n}}\right)=H(X)$ and $h\left(S_{t_{1}}, \ldots, S_{t_{n}}\right)=H(S)$.

So, let $h:[0, \infty)^{n} \rightarrow[0, \infty)$ be the payoff function for the path-dependent claim $H$. At times $t \in\left[0, t_{1}\right)$, the value function of $H(S)$ will be of the form

$$
v(t, x)=\mathrm{E}\left[H(S) \mid S_{t}=x\right]=\mathrm{E}\left[h\left(S_{t_{1}}, \ldots, S_{t_{n}}\right) \mid S_{t}=x\right] .
$$

By taking $t=0$ and $x=X_{0}$, this gives the price of the derivative in the local volatility model:

$$
v\left(0, X_{0}\right)=\mathrm{E}\left[H(S) \mid S_{0}=X_{0}\right] .
$$

At a later stage, for $t \in\left[t_{k}, t_{k+1}\right)$, the fixings $X_{t_{1}}, \ldots, X_{t_{k}}$ of the observed market prices will have been locked in as additional parameters of the value function:

$$
\begin{aligned}
v\left(t, X_{t_{1}}, \ldots, X_{t_{k}}, x\right) & =\mathrm{E}\left[H(S) \mid S_{t_{1}}=X_{t_{1}}, \ldots, S_{t_{k}}=X_{t_{k}}, S_{t}=x\right] \\
& =\mathrm{E}\left[h\left(X_{t_{1}}, \ldots, X_{t_{k}}, S_{t_{k+1}}, \ldots, S_{t_{n}}\right) \mid S_{t}=x\right]
\end{aligned}
$$


Here, the second identity follows from the Markov property of $S$. For $t \geq t_{n}$, we will finally have

$$
v\left(t, X_{t_{1}}, \ldots, X_{t_{n}}, x\right)=h\left(X_{t_{1}}, \ldots, X_{t_{n}}\right)=H(X) \text { for all } x .
$$

In analogy to our notation $H(X)$, we will use the shorthand notation

$$
v(t, X):=v\left(t, X_{t_{1}}, \ldots, X_{t_{k}}, X_{t}\right) \quad \text { for } t_{k} \leq t<t_{k+1},
$$

for the path-dependent value function. Similarly, we define $v(t, S)$, and we will use the same notation on other functions such as the derivatives of $v$.

Note that the local volatility model is a complete market model. Thus, the option's payoff in the local volatility model can be represented in terms of the corresponding delta hedging strategy:

$$
H(S)=v\left(0, S_{0}\right)+\int_{0}^{T} v_{x}(t, S) \mathrm{d} S_{t} \quad \text { P-a.s. }
$$

Here and in the sequel,

$$
v_{x}(t, S)=v_{x}\left(t, S_{t_{1}}, \ldots, S_{t_{k}}, S_{t}\right) \text { for } t_{k} \leq t<t_{k+1},
$$

will be shorthand for the derivative of the value function $v\left(t, x_{1}, \ldots, x_{k}, x\right)$ with respect to its final argument $x$, and we assume that this derivative is well defined for almost every (a.e.) $t$. Similarly, we will use the notations $v_{x x}$ and $v_{t}$.

The question we are interested in here is whether hedging with the delta obtained from the local volatility model also works for the physical price process $X$. That is, can we hedge the physical payoff $H(X)$ by replacing in (2.6) and (2.7) the model process $\left(S_{t}\right)$ with the real-world prices $\left(X_{t}\right)_{0 \leq t \leq T}$ ?

The corresponding self-financing hedging strategy will then involve the price $v\left(0, X_{0}\right)$, and the number of shares held at time $t$ will be determined by $v_{x}(t, X)$. If trading occurs at finitely many times $s_{1}<\cdots<s_{k}$ taken from a partition $\zeta_{n}=\left\{0, s_{1}, \ldots, s_{k}\right\} \subset[0, T]$ then the corresponding value process will be

$$
v\left(0, X_{0}\right)+\sum_{s_{i} \in \zeta_{n}} v_{x}\left(s_{i-1}, X\right)\left(X_{s_{i}}-X_{s_{i-1}}\right) .
$$

If we can pass to a limit in (2.8) for a fixed sequence $\zeta_{1} \subset \zeta_{2} \subset \cdots$ of partitions whose meshes tend to 0 then the value process takes the form

$$
v\left(0, X_{0}\right)+\int_{0}^{T} v_{x}(t, X) \mathrm{d} X_{t},
$$

where the integral on the right is the pathwise Itô integral defined in the sense of Föllmer [9]:

$$
\int_{0}^{T} v_{x}(t, X) \mathrm{d} X_{t}=\lim _{n \uparrow \infty} \sum_{s_{i} \in \zeta_{n}} v_{x}\left(s_{i-1}, X\right)\left(X_{s_{i}}-X_{s_{i-1}}\right) .
$$

According to Föllmer [9], this passage to the limit is possible if the path $t \mapsto X_{t}$ is continuous, has a continuous pathwise quadratic variation,

$$
\langle X\rangle_{t}:=\lim _{n \uparrow \infty} \sum_{\substack{s_{i} \in \zeta_{n} \\ s_{i} \leq t}}\left(X_{s_{i}}-X_{s_{i-1}}\right)^{2}
$$


and the value function $v$ is sufficiently regular. Thus, we will henceforth assume that $X$ satisfies the above conditions, and we point out once more that no stochastic model for $X$ is needed. Note that the above conditions are always satisfied if we take for $X$ a typical sample path of a continuous semimartingale. Conversely, not every path $X$ satisfying our assumptions has to come from a continuous semimartingale (this follows, for example, from Theorem 1.7 of [4] with $H=\frac{3}{4}$ ).

The main regularity conditions for $v$ can be deduced from the following regularity assumptions on the local volatility function and the payoff function $h$; see Proposition 2.1, below. These assumptions are almost identical to the ones in [8]. Some of them can be relaxed at the expense of tightening others; see the Remark at the end of this section. Since local volatility functions, in practice, typically arise as the interpolation of discrete values obtained from a discretized version of Dupire's formula, there is no loss of generality from a practical point of view in assuming smoothness and boundedness of our local volatility function.

Assumption 2.1. Throughout this paper we will assume that the local volatility function $\sigma(t, x)$ satisfies the following conditions:

(a) $\sigma:[0, T] \times(0, \infty) \rightarrow(0, \infty)$ is continuously differentiable, bounded, and bounded away from 0 ;

(b) $\sigma(t, x) x$ is Lipschitz continuous in $x$, uniformly in $t \in[0, T]$.

We will also henceforth assume that $h$ denotes a continuous function from $[0, \infty)^{n}$ to $[0, \infty)$ that satisfies the polynomial growth condition

$$
h(x) \leq C\left(1+|x|^{p}\right), \quad x \in[0, \infty)^{n},
$$

for certain constants $C, p \geq 0$. Such a function will be called a payoff function.

Remark. Assumption 2.1 has some immediate consequences. Firstly, the boundedness of $\sigma$ implies, via Novikov's theorem, that $S$ is a strictly positive martingale with finite moments $\mathrm{E}\left[S_{t}^{p} \mid S_{0}=x\right]$ for all $p \in[0, \infty)$. In particular, the value function associated with any payoff function $h$ is finite. Secondly, condition (b) ensures that the stochastic differential equation $\mathrm{d} S_{t}=\sigma\left(t, S_{t}\right) S_{t} \mathrm{~d} W_{t}$ admits a strong solution, which is pathwise unique, a property which is also important in practice to secure the convergence of numerical algorithms.

As long as the intermediate arguments $X_{t_{1}}, \ldots, X_{t_{k}}$ do not matter, we may only spell out the dependence of $v$ (and its derivatives) on its first and last arguments $t$ and $x$ by using the shorthand notation

$$
v(t, \cdot, x):=v(t, \underbrace{., \ldots, .}_{k \text { arguments }}, x) \text { for } t_{k} \leq t<t_{k+1} .
$$

We turn to a first result, which states the technical regularity properties of our value function needed for the definition of the delta hedging strategy.

Proposition 2.1. (Regularity of the value function.) Suppose that $h$ is a payoff function. Then $(t, x) \mapsto v(t, \cdot, x)$ is continuous on $[0, T] \times[0, \infty)$, continuously differentiable in $t \in \bigcup_{k}\left(t_{k}, t_{k+1}\right)$, twice continuously differentiable in $x \in(0, \infty)$, and satisfies the partial differential equation

$$
v_{t}(t, \cdot, x)+\frac{1}{2} \sigma(t, x)^{2} x^{2} v_{x x}(t, \cdot, x)=0, \quad t \in \bigcup_{k}\left(t_{k}, t_{k+1}\right), x \in(0, \infty) .
$$


Moreover, if

$$
\int_{0}^{T}\left|v_{x x}(t, X)\right| \mathrm{d}\langle X\rangle_{t}+\int_{0}^{T}\left|v_{t}(t, X)\right| \mathrm{d} t<\infty
$$

then the pathwise Itô integral $\int_{0}^{T} v_{x}(t, X) \mathrm{d} X_{t}$ is well defined, and Itô's formula holds:

$$
v(T, X)=v(0, X)+\int_{0}^{T} v_{x}(t, X) \mathrm{d} X_{t}+\frac{1}{2} \int_{0}^{T} v_{x x}(t, X) \mathrm{d}\langle X\rangle_{t}+\int_{0}^{T} v_{t}(t, X) \mathrm{d} t .
$$

Condition (2.10) is clearly necessary to make sense of the right-hand side of (2.11) and in turn of the delta hedging strategy. It is satisfied as soon as $h$ belongs to $C^{2}$.

Let us return to our problem of hedging the physical payoff $H(X)$ of a path-dependent option with the delta hedging strategy obtained from the local volatility model. The trader carrying out the hedge will insert at each time $t$ the market spot price $X_{t}$ into the local volatility model. In particular, $\sigma\left(t, X_{t}\right)$ will serve as an estimate for the short volatility at time $t$. Thus, we will say that $\sigma$ overestimates the market volatility if

$$
\int_{s}^{t} \sigma^{2}\left(r, X_{r}\right) X_{r}^{2} \mathrm{~d} r \geq\langle X\rangle_{t}-\langle X\rangle_{s} \text { for all } 0 \leq s<t \leq T .
$$

If the reverse inequality holds, we will say that $\sigma$ underestimates the market volatility.

Remark. If inequality (2.12) holds then the function $t \mapsto\langle X\rangle_{t}$ is absolutely continuous and can hence be written as $\langle X\rangle_{t}=\int_{0}^{t} \varsigma_{s} X_{s}^{2} \mathrm{~d} s$ for some function $\varsigma \geq 0$, which can be interpreted as the short variance of the market. In this case, (2.12) is equivalent to requiring that

$$
\sigma\left(t, X_{t}\right) \geq \sqrt{\varsigma_{t}} \text { for a.e. } t \in[0, T] \text { such that } X_{t}>0 .
$$

Definition. (Robustness of the delta hedging strategy.) Let $H(X)=h\left(X_{t_{1}}, \ldots, X_{t_{n}}\right)$ be the payoff of a path-dependent derivative satisfying (2.10). We will say that the delta hedging strategy for $H$ obtained from the local volatility model is robust if the following two conditions hold. If $\sigma$ overestimates the market volatility then there is a superhedge for the seller in the sense that

$$
v\left(0, X_{0}\right)+\int_{0}^{T} v_{x}(t, X) \mathrm{d} X_{t} \geq H(X) .
$$

If $\sigma$ underestimates the market volatility then there is a superhedge for the buyer in the sense that

$$
v\left(0, X_{0}\right)+\int_{0}^{T} v_{x}(t, X) \mathrm{d} X_{t} \leq H(X) .
$$

The preceding notion of robustness is due to El Karoui et al. [8]. If a delta hedging strategy is robust then a trader can monitor its performance by comparing $\sigma\left(t, X_{t}\right)$ to the realized market volatility.

Remark. (Volatility comparison for prices.) While our notion of robustness is understood in a strictly pathwise sense, some other authors emphasize its impact on option pricing rather than hedging. To this end, we assume that the market prices $X$ are a particular realization of the sample paths of a continuous-local martingale. Then, if $\sigma$ overestimates the market volatility almost surely and if the delta hedging strategy is a supermartingale, taking expectations in (2.13) yields

$$
v\left(0, X_{0}\right)=\mathrm{E}\left[H(S) \mid S_{0}=X_{0}\right] \geq \mathrm{E}[H(X)] .
$$


This result can be interpreted as an ordering between the price computed in the local volatility model and the 'true' market price of the derivative. This latter price, however, is not observable unless the derivative is liquidly traded.

We can now state our first basic hedging result. It relates the robustness of the delta hedging strategy to the positivity of the corresponding gamma, $v_{x x}$. Note that the positivity of the gamma will follow automatically if the function $x \mapsto v(t, \cdot, x)$ is convex for all $t$.

Proposition 2.2. (Positivity of the gamma implies robustness.) Suppose that $h$ is a payoff function such that the option's gamma satisfies $v_{x x}(t, X) \geq 0$ for a.e. $t$ and such that (2.10) holds. Then the delta hedging strategy is robust.

Proof. The proof is short and instructive. It relies on arguments from [8]. By Proposition 2.1 and since $v(T, X)=H(X)$,

$$
v(0, X)+\int_{0}^{T} v_{x}(t, X) \mathrm{d} X_{t}=H(X)-\frac{1}{2} \int_{0}^{T} v_{x x}(t, X) \mathrm{d}\langle X\rangle_{t}-\int_{0}^{T} v_{t}(t, X) \mathrm{d} t .
$$

Now suppose that $\sigma$ overestimates the market volatility. If $v_{x x}(t, X) \geq 0$ then we obtain $\int_{0}^{T} v_{x x}(t, X) \mathrm{d}\langle X\rangle_{t} \leq \int_{0}^{T} v_{x x}(t, X) \sigma\left(t, X_{t}\right)^{2} X_{t}^{2} \mathrm{~d} t$. The partial differential equation (PDE), (2.9), then implies that the right-hand side of (2.14) dominates $H(X)$, i.e. we have a successful seller's hedge. The argument for the buyer's hedge is identical.

Our next result shows that the delta hedging strategy obtained from a Black-Scholes model will be robust for all convex payoff functions. This class includes, in particular, all Asian options, lookback puts, floating-strike forward starts, and their aggregated cliquets. Perhaps this fact might help to explain the good hedging performance of the Black-Scholes model, which is sometimes reported by practitioners.

Corollary 2.1. (Robustness in a Black-Scholes model.) Suppose that the local volatility function $\sigma(t, x)$ does not depend on $x$. Then the value function of any convex payoff function is again convex. In particular, the corresponding delta hedging strategy is robust if (2.10) holds.

In a genuine local volatility model it is known, from [8], that all European-style derivatives $h\left(S_{T}\right)$ with a convex payoff function $h$ have convex payoff functions and thus admit robust delta hedges. For path-dependent derivatives, however, the situation is more complicated and less stable. The value functions of a large number of fairly standard path-dependent derivatives may not be convex and delta hedging may not be robust, even though they correspond to convex payoff functions. This is illustrated by our next result. Its conditions in particular apply to average-strike Asian options, lookback puts, floating-strike forward starts, and their aggregated cliquets.

Theorem 2.1. (Nonrobustness for standard derivatives.) Suppose that $h$ is a convex payoff function that is not identically equal to a linear functional and is positively homogeneous:

$$
h\left(z x_{1}, \ldots, z x_{n}\right)=z h\left(x_{1}, \ldots, x_{n}\right) \text { for } z \geq 0 .
$$

Moreover, suppose that there exist constants $0<c<C$ and $0<x_{0}<x_{1}$ such that the local volatility function $\sigma(\cdot)$ satisfies $\sigma(t, x) \geq C$ if $0 \leq x \leq x_{0}$ and $\sigma(t, x) \leq c$ if $x_{1} \leq x<\infty$. Then the corresponding value function is not convex and there exists a path $X$ along which the delta hedging strategy is not robust. 
In Theorem 2.1 the particular shape of $\sigma(t, x)$ for $x_{0}<x<x_{1}$ can be arbitrary. The assumed property is in fact a frequently observed empirical pattern of local volatility functions in many equity markets.

Our final result gives a sufficient condition on a payoff function $h$ guaranteeing that the corresponding value function is convex in its final argument for any local volatility model. Thus, the delta hedging strategy of such a payoff will be robust. Our condition is the directional convexity of $h$, that is, all marginal functions $x_{i} \mapsto h\left(x_{1}, \ldots, x_{i}, \ldots, x_{n}\right)$ are convex and all right-hand derivatives

$$
h_{x_{i}}^{+}\left(x_{1}, \ldots, x_{n}\right):=\lim _{\delta \downarrow 0} \frac{1}{\delta}\left(h\left(x_{1}, \ldots, x_{i}+\delta, \ldots, x_{n}\right)-h\left(x_{1}, \ldots, x_{i}, \ldots, x_{n}\right)\right)
$$

are increasing with respect to each component $x_{j}$. Within our example class (2.1)-(2.5), this condition is satisfied by the average-price Asian call options,

$$
\left(\frac{1}{n} \sum_{i=0}^{n} S_{t_{i}}-K\right)^{+}
$$

Note that directional convexity neither implies nor is implied by convexity in the usual sense: there are nonconvex but directionally convex payoff functions such as the one corresponding to a 'geometric Asian call',

$$
\left(\prod_{i=0}^{n} S_{t_{i}}-K\right)^{+}
$$

Theorem 2.2. (Directional convexity implies robustness.) Let $h$ be a directionally convex payoff function. Then, for $t_{k-1} \leq t<t_{k}$, the value function $v\left(t, x_{1}, \ldots, x_{k}\right)$ is also directionally convex in $x_{1}, \ldots, x_{k}$. In particular, $x \mapsto v(t, \cdot, x)$ is convex and the corresponding delta hedging strategy is robust as soon as (2.10) holds.

Remark. (On the standard definition of directional convexity.) The standard definition of directional convexity of a function $f: \mathbb{R}^{n} \rightarrow \mathbb{R}$ requires that

$$
x_{j} \mapsto f\left(x_{1}, \ldots, x_{i}+\varepsilon, \ldots, x_{n}\right)-f\left(x_{1}, \ldots, x_{i}, \ldots, x_{n}\right)
$$

is an increasing function whenever $\varepsilon>0$ and $i, j \in\{1, \ldots, n\}$; see [1] and the references therein. Taking $j=i$ implies that the function $g\left(x_{i}\right):=f\left(x_{1}, \ldots, x_{i}, \ldots, x_{n}\right)$ is Wright convex, i.e. $x \mapsto g(x+\varepsilon)-g(x)$ is increasing for each $\varepsilon>0$. As observed by Wright [24], this immediately implies that

$$
g(x)+g(y) \geq 2 g\left(\frac{x+y}{2}\right) .
$$

It is well known that the preceding inequality implies convexity if $g$ is continuous; see, e.g. [16, Theorem 86]. Hence, we can conclude that our definition of directional convexity coincides with the usual one as soon as $x_{i} \mapsto f\left(x_{1}, \ldots, x_{i}, \ldots, x_{n}\right)$ is continuous for each $i$. However, Hardy et al. [16, p. 96] constructed a discontinuous function $g$ such that $g(x+y)=g(x)+g(y)$. Such a discontinuous but additive $g$ is clearly Wright convex but not convex in the usual sense. Thus, our definition of directional convexity is generally stronger than the standard one. 
Remark. (Positive homogeneity excludes directional convexity.) Combining Theorems 2.1 and 2.2 shows that every convex payoff function $h$ that is both positively homogeneous and directionally convex must be linear. In fact, the additional assumption of convexity can be dropped as is shown by the following direct argument. For $n=2$ and $x_{1}, x_{2}>0$, positive homogeneity yields

$$
\frac{h\left(x_{1}+t, x_{2}\right)-h\left(x_{1}, x_{2}\right)}{t}=\frac{h\left(x_{1} / x_{2}+t / x_{2}, 1\right)-h\left(x_{1} / x_{2}, 1\right)}{t / x_{2}} .
$$

Letting $t \downarrow 0$ yields

$$
h_{x_{1}}^{+}\left(x_{1}, x_{2}\right)=h_{x_{1}}^{+}\left(\frac{x_{1}}{x_{2}}, 1\right) .
$$

Since $h$ is directionally convex, the left-hand side is increasing and the right-hand side is decreasing in $x_{2}$. Thus, $h_{x_{1}}^{+}\left(x_{1}, x_{2}\right)$ is independent of $x_{2}$, and the continuity and positive homogeneity of $h$ imply that

$$
\begin{aligned}
h\left(x_{1}, x_{2}\right) & =h\left(0, x_{2}\right)+\int_{0}^{x_{1}} h_{x_{1}}^{+}\left(u, x_{2}\right) \mathrm{d} u \\
& =h\left(0, x_{2}\right)+h\left(x_{1}, 0\right)+h(0,0) \\
& =x_{2} h(0,1)+x_{1} h(1,0),
\end{aligned}
$$

i.e. $h$ is linear. For $n>2$, we can use induction and Lemma 3.3, below.

Remark. (Recalibration.) Our approach assumes that the local volatility model is set up at time $t=0$ and not altered in the sequel. In practice, market models sometimes undergo a recalibration process during which model parameters are refitted at intermediate times. In our case, this would mean that the volatility function $\sigma(t, x)$ would become dependent in a highly nontrivial way on the prices of the underlying and all plain-vanilla options observable at time $t$. Recalibration will therefore considerably complicate the situation. It is interesting to note that recalibration may in fact destabilize pricing and hedging of derivatives; see [2] and [3] for a recent analysis of parameter recalibration in stochastic volatility models.

Remark. (Alternative conditions on $\sigma$ and $h$.) Instead of Assumption 2.1 we could also work with the following set of conditions:

(i) $\sigma:[0, T] \times(0, \infty) \rightarrow(0, \infty)$ is continuous, bounded, locally Hölder continuous in $(t, x)$, and continuously differentiable with respect to $x$;

(ii) $\partial \sigma(t, x) / \partial x$ is locally Hölder continuous in $(t, x)$, and there exists some constant $A>0$ such that

$$
\left|\frac{\partial}{\partial x} \sigma(t, x)\right| \leq A \frac{\log (1+x)}{1+x} .
$$

Moreover, suppose that the payoff function $h\left(x_{1}, \ldots, x_{n}\right)$ is continuously differentiable and its partial derivatives grow at most logarithmically. If these conditions are satisfied then variants of Proposition 2.1 and Theorem 2.2 hold. In addition, the option's delta $v_{x}(t, \cdot, x)$ grows at most logarithmically in $x$; see [23]. 
Remark. (Possible extensions.) It is an interesting question whether robustness or nonrobustness of hedging strategies hold for other classes of price processes than the ones studied here. For multivariate local volatility models, Bergenthum and Rüschendorf [1] provided sufficient conditions on the local volatility matrix under which prices of European-style derivatives $H(S)=h\left(S_{T}\right)$ with $h$ convex are again convex functions of $S_{0}$. Ekström et al. [7], conversely, singled out a class of volatility matrices for which prices of European-style derivatives may not be convex and robustness fails. If we stay within the class of complete Itô diffusion models, but allow volatility to be path-dependent in a general way, then prices of standard European call options may not be convex, as is shown by a one-dimensional example in [8, Section 4]. Other model classes used in practice are often incomplete. So, while Bergenthum and Rüschendorf [1] established convexity of European option prices in certain models based on Lévy processes, there is typically no associated hedging strategy for which robustness could be investigated. A similar situation occurs in classical stochastic volatility models such as the Heston model: since volatility is not a traded asset, delta hedging is usually not sufficient to replicate an option. In practice, stochastic volatility models are therefore often completed by adding liquidly traded derivatives such as variance swaps or call options. This leads to multivariate models of local volatility-type in which options admit so-called delta-vega hedges; see, e.g. [2], [3], and [5]. However, the completed models normally do not satisfy the assumptions of [1] or [7], so that even the robustness of delta-vega hedges for European-style derivatives is not a priori clear.

\section{Proofs}

We recall that we work under the conditions of Assumption 2.1. The following lemma relies on standard facts of parabolic partial differential equations.

Lemma 3.1. Let $h:[0, \infty) \rightarrow(0, \infty)$ be a payoff function. Then $v(t, x):=\mathrm{E}\left[h\left(S_{T}\right) \mid S_{t}=\right.$ $x]$ belongs to $C([0, T] \times[0, \infty)) \cap C^{1,2}([0, T) \times(0, \infty))$, satisfies a polynomial growth condition uniformly in $t \in[0, T]$, and solves the Cauchy problem

$$
v_{t}(t, x)+\frac{1}{2} \sigma^{2}(t, x) x^{2} v_{x x}(t, x)=0 \quad \text { in }(0, T) \times(0, \infty)
$$

and

$$
v(T, x)=h(x) .
$$

Proof. According to [19, Theorem A.14], the above Cauchy problem has a unique solution $v \in C([0, T] \times[0, \infty)) \cap C^{1,2}([0, T) \times(0, \infty))$ with polynomial growth. Thus, the assertion follows from [20, Theorem 5.7.6].

Proof of Proposition 2.1. The idea is to apply Lemma 3.1 and backward induction. To this end, let us introduce the dummy variables $x_{1}, \ldots, x_{n-1} \in(0, \infty)$ and the function $h_{1}(x):=$ $h\left(x_{1}, \ldots, x_{n-1}, x\right)$. For $t_{n-1} \leq t<t_{n}$, we can directly apply Lemma 3.1 and conclude that $v\left(t, x_{1}, \ldots, x_{n-1}, x\right)=\mathrm{E}\left[h_{1}\left(S_{t_{n}}\right) \mid S_{t}=x\right]$ satisfies the regularity conditions and the PDE in question.

For the case in which $t_{n-2} \leq t<t_{n-1}$, we define

$$
h_{2}(x):=\mathrm{E}\left[h\left(x_{1}, \ldots, x_{n-2}, x, S_{t_{n}}\right) \mid S_{t_{n-1}}=x\right] .
$$

Then by the Markov property of $S$ we have

$$
\begin{aligned}
v\left(t, x_{1}, \ldots, x_{n-2}, x\right) & =\mathrm{E}\left[h\left(x_{1}, \ldots, x_{n-2}, S_{t_{n-1}}, S_{t_{n}}\right) \mid S_{t}=x\right] \\
& =\mathrm{E}\left[h_{2}\left(S_{t_{n-1}}\right) \mid S_{t}=x\right] .
\end{aligned}
$$


Hence, the assertion will follow for $t_{n-2} \leq t<t_{n-1}$ by Lemma 3.1 if we can show that $h_{2}$ is again a payoff function. Let us first show the polynomial growth condition. Owing to the polynomial growth condition for $h$, there exist constants $C, p \geq 1$ such that

$$
h\left(x_{1}, \ldots, x_{n-2}, x, S_{t_{n}}\right) \leq C\left(1+x_{1}^{p}+\cdots+x_{n-2}^{p}+x^{p}+S_{t_{n}}^{p}\right) .
$$

Thus, according to Lemma 3.1, $h_{2}$ also satisfies the polynomial growth condition. Regarding the proof of the continuity of $h_{2}$, let $\widetilde{h}(y, z):=h\left(x_{1}, \ldots, x_{n-2}, y, z\right)$. Then

$$
\begin{aligned}
\left|h_{2}(x)-h_{2}(y)\right| \leq & \left|\mathrm{E}\left[\tilde{h}\left(x, S_{t_{n}}\right) \mid S_{t_{n-1}}=x\right]-\mathrm{E}\left[\tilde{h}\left(x, S_{t_{n}}\right) \mid S_{t_{n-1}}=y\right]\right| \\
& +\left|\mathrm{E}\left[\tilde{h}\left(x, S_{t_{n}}\right)-\tilde{h}\left(y, S_{t_{n}}\right) \mid S_{t_{n-1}}=y\right]\right| .
\end{aligned}
$$

As $x \rightarrow y$, the first difference on the right tends to 0 by Lemma 3.1. For the second difference, we can use (3.1) and dominated convergence (see the Remark following Assumption 2.1). Thus, $h_{2}$ is also continuous. Backward induction on $n$ concludes the proof of the regularity assertions of $v$ and of (2.9).

We now prove Itô's formula, (2.11). Owing to the established regularity properties of $v$, we may apply Föllmer's pathwise Itô formula [9] for $t_{k}<s<t<t_{k+1}$ :

$$
v(t, X)=v(s, X)+\int_{s}^{t} v_{x}(r, X) \mathrm{d} X_{r}+\frac{1}{2} \int_{s}^{t} v_{x x}(r, X) \mathrm{d}\langle X\rangle_{r}+\int_{s}^{t} v_{t}(r, X) \mathrm{d} r .
$$

Since $v$ is continuous and (2.10) holds, we may pass to the limit as $s \downarrow t_{k}$ and $t \uparrow t_{k+1}$. Summing over $k$ then yields (2.11).

Proof of Corollary 2.1. Owing to Propositions 2.1 and 2.2, we have only to prove the convexity of $x \mapsto v(t, \cdot, x)$. However, this follows immediately from the fact that the path of a geometric Brownian motion with time-dependent volatility $\sigma(t)$ and $S_{0}=x$ is given by

$$
S_{t}=x \exp \left(\int_{0}^{t} \sigma(s) \mathrm{d} W_{s}-\frac{1}{2} \int_{0}^{t} \sigma^{2}(s) \mathrm{d} s\right), \quad t \geq 0,
$$

and thus is affine in its starting point $x$.

Now we will prepare for the proof of Theorem 2.1. We denote by $S_{t}^{\gamma}$ the price process in a Black-Scholes model with constant volatility $\gamma>0$. More precisely,

$$
S_{t}^{\gamma}=S_{0} \exp \left(\gamma W_{t}-\frac{1}{2} \gamma^{2} t\right)
$$

We write $v^{\gamma}$ for the value function corresponding to $H\left(S^{\gamma}\right)$. Note that if $h$ is positively homogeneous then so is $v^{\gamma}(t, \cdot)$.

Lemma 3.2. For $S_{0}^{\gamma}=1$, the function $\gamma \mapsto \mathrm{E}\left[H\left(S^{\gamma}\right)\right]$ is strictly increasing if $h$ satisfies the assumptions of Theorem 2.1.

Proof. We proceed by induction on $n$. For $n=1$, the convex function $h$ can be represented as

$$
h(x)=h(0)+h^{\prime}(0+) x+\int_{(0, \infty)}(x-K)^{+} \mu(\mathrm{d} K)
$$

for a positive Radon measure $\mu$ on $(0, \infty)$, where $h^{\prime}(0+)$ denotes the right-hand derivative of $h$ at 0 ; see, e.g. [12, Example 1.24]. This measure $\mu$ must be nonzero since $h$ is not linear. 
Fubini's theorem thus yields, for all $t, \gamma>0$,

$$
\mathrm{E}\left[h\left(S_{t}^{\gamma}\right)\right]=h(0)+h^{\prime}(0+)+\int_{(0, \infty)} \mathrm{E}\left[\left(S_{t}^{\gamma}-K\right)^{+}\right] \mu(\mathrm{d} K) .
$$

It follows, from the standard Black-Scholes formula, that $\gamma \mapsto \mathrm{E}\left[\left(S_{t}^{\gamma}-K\right)^{+}\right]$is strictly increasing, and this proves our claim for $n=1$. Now suppose that our claim has already been established for a given $n \geq 1$ and that $h\left(x_{1}, \ldots, x_{n+1}\right)$ is a convex payoff function, which is not linear. If $\left(x_{2}, \ldots, x_{n+1}\right) \mapsto h\left(x_{1}, x_{2}, \ldots, x_{n+1}\right)$ is linear for $x_{1}=1$ and hence for all $x_{1}>0$, then we are back to the situation where $n=1$. Otherwise, the induction hypothesis and the Markov property of $S^{\gamma}$ imply that

$$
\gamma \longmapsto \mathrm{E}\left[h\left(1, S_{t_{2}-t_{1}}^{\gamma}, \ldots, S_{t_{n+1}-t_{1}}^{\gamma}\right)\right]=\frac{1}{x} v^{\gamma}\left(t_{1}, x, x\right)
$$

is strictly increasing for all $x>0$. Thus, we find that, for $\gamma<\kappa$,

$$
\mathrm{E}\left[H\left(S^{\gamma}\right)\right]=\mathrm{E}\left[v^{\gamma}\left(t_{1}, S_{t_{1}}^{\gamma}, S_{t_{1}}^{\gamma}\right)\right]=v^{\gamma}\left(t_{1}, 1,1\right)<v^{\kappa}\left(t_{1}, 1,1\right)=\mathrm{E}\left[H\left(S^{\kappa}\right)\right] .
$$

This concludes the proof.

Proof of Theorem 2.1. We will show that $v(0, x)=\mathrm{E}_{x}[H(S)]$ is not convex, where the subscript $x$ indicates that we are considering the model with $S_{0}=x$. To this end, it is enough to show that

$$
\liminf _{x \downarrow 0} \frac{v(0, x)}{x}>\limsup _{y \uparrow \infty} \frac{v(0, y)}{y} .
$$

Let $\bar{\sigma}(t, x):=C \vee \sigma(t, x)$, where $a \vee b=\max (a, b)$, and consider the solution $X$ of the stochastic differential equation (SDE)

$$
\mathrm{d} X_{t}=\bar{\sigma}\left(t, X_{t}\right) X_{t} \mathrm{~d} W_{t}, \quad X_{0}=S_{0} .
$$

Owing to the pathwise uniqueness of our SDEs, the sample paths of $S$ and $X$ coincide up to

$$
\tau:=\inf \left\{t \geq 0 \mid \sigma\left(t, S_{t}\right) \leq C\right\} .
$$

Moreover, $C$ underestimates the 'market volatility' of $X$, i.e. $C^{2} X_{t}^{2} \mathrm{~d} t \leq \bar{\sigma}\left(t, X_{t}\right)^{2} \mathrm{~d} t=\mathrm{d}\langle X\rangle_{t}$, and Corollary 2.1 yields

$$
v^{C}\left(0, X_{0}\right)+\int_{0}^{T} v_{x}^{C}(s, X) \mathrm{d} X_{s} \leq H(X) .
$$

Since $\sigma$ is bounded from above, $X$ is a true martingale owing to Novikov's theorem. Moreover, it follows, from the positive homogeneity and continuity of $h$, that $v^{C}(t, \cdot, x)$ grows at most linearly in $x$. Hence, $v_{x}^{C}(s, X)$ is bounded for a.e. $s$, and it follows that $\int v_{x}^{C}(s, X) \mathrm{d} X_{s}$ is a true martingale. Taking expectations thus yields

$$
\mathrm{E}_{x}[H(X)] \geq v^{C}(0, x)=x v^{C}(0,1) .
$$

Thus,

$$
\begin{aligned}
v(0, x) & =\mathrm{E}_{x}[H(S)] \\
& \geq \mathrm{E}_{x}[H(S) ; \tau \geq T] \\
& =\mathrm{E}_{x}[H(X) ; \tau \geq T] \\
& \geq x v^{C}(0,1)-\mathrm{E}_{x}[H(X) ; \tau<T] .
\end{aligned}
$$


We will show below that

$$
\frac{1}{x} \mathrm{E}_{x}[H(X) ; \tau<T] \longrightarrow 0 \quad \text { as } x \downarrow 0 .
$$

From this, it follows that

$$
\liminf _{x \downarrow 0} \frac{v(0, x)}{x} \geq v^{C}(0,1)
$$

Similarly, we can show that

$$
\limsup _{y \uparrow \infty} \frac{v(0, y)}{y} \leq v^{c}(0,1),
$$

and this completes the proof of (3.3) when combined with Lemma 3.2.

To prove (3.4), first note that there exists a constant $k \geq 0$ such that $H(X) \leq k \max _{t} X_{t}$, owing to the continuity and positive homogeneity of $h$. Writing $X_{t}=x M_{t}$, where the martingale $M$ is the Doleans-Dade exponential $\mathcal{E}\left(\int_{0} \bar{\sigma}\left(t, X_{t}\right) \mathrm{d} W_{t}\right)$, we see that

$$
\frac{1}{x} \mathrm{E}_{x}[H(X) ; \tau<T] \leq k \mathrm{E}_{x}\left[\max _{t \leq T} M_{t} ; \tau<T\right] \leq k \mathrm{E}_{x}\left[\max _{t \leq T} M_{t}^{2}\right]^{1 / 2} \mathrm{P}_{x}[\tau<T]^{1 / 2} .
$$

Next, by Doob's inequality,

$$
\mathrm{E}_{x}\left[\max _{t \leq T} M_{t}^{2}\right] \leq 2 \mathrm{E}_{x}\left[M_{T}^{2}\right] \leq 2 \mathrm{e}^{\Sigma T},
$$

where $\Sigma$ is an upper bound for $\sigma$. Furthermore, $S$ is a true martingale so that

$$
x=\mathrm{E}_{x}\left[S_{\tau \wedge T}\right] \geq \mathrm{E}_{x}\left[S_{\tau \wedge T} ; \tau<T\right] \geq x_{0} \mathrm{P}_{x}[\tau<T] .
$$

Thus, $\mathrm{P}_{x}[\tau<T] \rightarrow 0$ as $x \downarrow 0$, and (3.4) follows.

It remains to construct a path $X$ along which the delta hedging strategy is not robust. Owing to the nonconvexity of $v(0, \cdot)$, there exists some $x>0$ such that $v_{x x}(0, x)<0$. Now let $X^{1}$ be a typical path of a geometric Brownian motion with a volatility $\gamma$ that is a strict lower bound for $\sigma(\cdot)$, and suppose that $X_{0}^{1}=x$. The continuity of $v_{x x}$ implies that

$$
\tau:=\inf \left\{t \geq 0 \mid v_{x x}\left(t, X_{t}^{1}\right) \geq 0\right\} \wedge \frac{t_{1}}{2}
$$

is strictly positive. Furthermore, let $X^{2}$ be a typical path of the solution to the SDE $\mathrm{d} S_{t}=$ $\sigma\left(t, S_{t}\right) S_{t} \mathrm{~d} W_{t}$ such that $X_{\tau}^{2}=X_{\tau}^{1}$. We claim that $X_{t}:=X_{t}^{1} \mathbf{1}_{\{t \leq \tau\}}+X_{t}^{2} \mathbf{1}_{\{t>\tau\}}$ does the job. First note that $\mathrm{d}\langle X\rangle_{t}=\gamma^{2} X_{t}^{2} \mathrm{~d} t<\sigma^{2}\left(t, X_{t}\right) X^{2} \mathrm{~d} t$ for $t \leq \tau$ and $\mathrm{d}\langle X\rangle_{t}=\sigma^{2}\left(t, X_{t}\right) X^{2} \mathrm{~d} t$ for $t>\tau$, so that $\sigma$ overestimates the volatility of $X$. Moreover, we have

$$
v\left(0, S_{0}\right)+\int_{0}^{\tau} v_{x}\left(t, S_{t}\right) \mathrm{d} S_{t}=v\left(\tau, S_{\tau}\right) \quad \text { and } \quad v\left(\tau, X_{\tau}\right)+\int_{\tau}^{T} v_{x}(\tau, X) \mathrm{d} X_{t}=H(X) .
$$

These Itô integrals are all well defined, owing to the martingale property of $v(t, S)$ and the martingale representation theorem for $S$. Using $v_{x x}\left(t, X_{t}\right)<0$ for $t<\tau$ and arguing as in the proof of Proposition 2.2, we obtain

$$
v\left(0, X_{0}\right)+\int_{0}^{\tau} v_{x}\left(t, X_{t}\right) \mathrm{d} X_{t}<v\left(\tau, X_{\tau}\right)
$$


and thus

$$
v\left(0, X_{0}\right)+\int_{0}^{T} v_{x}(t, X) \mathrm{d} X_{t}<H(X),
$$

i.e. the delta hedging strategy is not robust along the path $X$.

Let us now turn to the proof of Theorem 2.2. We prepare its proof with the following two auxiliary results.

Proposition 3.1. Suppose that $h\left(x_{1}, \ldots, x_{n}\right)$ is a directionally convex payoff function and $0 \leq$ $t<T$ are given. Then the function

$$
u\left(x_{1}, \ldots, x_{n}\right):=\mathrm{E}\left[h\left(x_{1}, \ldots, x_{n-1}, S_{T}\right) \mid S_{t}=x_{n}\right]
$$

is also directionally convex.

Proof. Owing to Proposition 2.1 and the Remark following Theorem 2.2, we have to show that

$$
x_{j} \longmapsto u\left(x_{1}, \ldots, x_{i}+\varepsilon, \ldots, x_{n}\right)-u\left(x_{1}, \ldots, x_{i}, \ldots, x_{n}\right)
$$

is increasing for all $\varepsilon>0$ and $i, j \in\{1, \ldots, n\}$. This property is clear for $i, j<n$. To prove it for the remaining cases, let $S_{T}^{t, x}$ denote the solution of $\mathrm{d} S_{r}=\sigma\left(r, S_{r}\right) S_{r} \mathrm{~d} W_{r}$ starting at time $t$ in $S_{t}^{t, x}=x$. Owing to a standard comparison result, we have $S_{T}^{t, y} \geq S_{T}^{t, x} \mathrm{P}$-a.s. for $y \geq x$; see, e.g. [20, Proposition 5.2.18]. Hence, (3.5) is increasing for $i<n$ and $j=n$ or for $i=n$ and $j<n$. For $i=j=n$, we have to show the convexity of $u$ in its final argument. For the case in which $h$ grows at most linearly in $x_{n}$, this follows from Theorem 5.2 of [8]. The general case can now be deduced by representing the convex function $x_{n} \mapsto h\left(x_{1}, \ldots, x_{n}\right)$ as in (3.2).

Lemma 3.3. If $h\left(x_{1}, \ldots, x_{n+1}\right)$ is directionally convex then so is the contraction

$$
g\left(x_{1}, \ldots, x_{n-1}, x_{n}\right):=h\left(x_{1}, \ldots, x_{n-1}, x_{n}, x_{n}\right) .
$$

Proof. Owing to the Remark following Theorem 2.2, we have to show that

$$
x_{j} \longmapsto g\left(x_{1}, \ldots, x_{i}+\varepsilon, \ldots, x_{n}\right)-g\left(x_{1}, \ldots, x_{i}, \ldots, x_{n}\right)
$$

is increasing for all $\varepsilon>0$ and $i, j \in\{1, \ldots, n\}$. This property is clear for $i<n$. If $i=n$ then

$$
\begin{aligned}
g\left(x_{1}, \ldots, x_{n-1}, x_{n}+\varepsilon\right)-g\left(x_{1}, \ldots, x_{n-1}, x_{n}\right) & \\
= & {\left[h\left(x_{1}, \ldots, x_{n}+\varepsilon, x_{n}+\varepsilon\right)-h\left(x_{1}, \ldots, x_{n}+\varepsilon, x_{n}\right)\right] } \\
& +\left[h\left(x_{1}, \ldots, x_{n}+\varepsilon, x_{n}\right)-h\left(x_{1}, \ldots, x_{n}, x_{n}\right)\right] .
\end{aligned}
$$

Clearly, either of the two terms in square brackets is increasing in any of the variables $x_{1}, \ldots, x_{n}$, and the result follows.

Proof of Theorem 2.2. Take an arbitrary time point $t_{n+1}>t_{n}$. We will prove our assertion for $t_{k} \leq t<t_{k+1}$ by backward induction on $k$. There is nothing to show if $k=n$. So let us suppose that the directional convexity of $v\left(t_{k+1}, x_{1}, \ldots, x_{k+2}\right)$ with respect to $x_{1}, \ldots, x_{k+2}$ has already been established and that $t_{k} \leq t<t_{k+1}$. Using the Markov property of $S$, we see that

$$
\begin{aligned}
v\left(t, x_{1}, \ldots, x_{k}, x\right) & =\mathrm{E}\left[v\left(t_{k+1}, x_{1}, \ldots, x_{k}, S_{t_{k+1}}, S_{t_{k+1}}\right) \mid S_{t}=x\right] \\
& =\mathrm{E}\left[g\left(x_{1}, \ldots, x_{k}, S_{t_{k+1}}\right) \mid S_{t}=x\right],
\end{aligned}
$$

where $g\left(x_{1}, \ldots, x_{k}, x_{k+1}\right):=v\left(t_{k+1}, x_{1}, \ldots, x_{k}, x_{k+1}, x_{k+1}\right)$. By Lemma 3.3 and the induction hypothesis, the function $g$ is directionally convex. Hence, the assertion follows from Proposition 3.1. 


\section{Acknowledgements}

It is a pleasure to thank Hans Bühler and Stephan Sturm for discussions. We also thank Antje Schulz for comments. This research was supported by Deutsche Forschungsgemeinschaft through the Research Center MATHEON ‘Mathematics for key technologies’ (FZT 86).

\section{References}

[1] Bergenthum, J. And RÜschendorf, L. (2006). Comparison of option prices in semimartingale models. Finance Stoch. 10, 222-249.

[2] BÜHLER, H. (2005). Volatility markets. Consistent modeling, hedging and practical implementation. Doctoral thesis, TU Berlin.

[3] BüHLER, H. (2006). Consistent variance swap models. Finance Stoch. 10, 178-203.

[4] Cheridito, P. (2001). Mixed fractional Brownian motion. Bernoulli 7, 913-934.

[5] Davis, M. (2004). Complete-market models of stochastic volatility. Proc. R. Soc. London A 460, 11-26.

[6] DupIRe, B. (1997). Pricing and hedging with smiles. In Mathematics of Derivative Securities (Cambridge, 1995; Publ. Newton Inst. 15), Cambridge University Press, pp. 103-111.

[7] Ekström, E., Janson, S. And Tysk, J. (2005). Superreplication of options on several underlying assets. J. Appl. Prob. 42, 27-38.

[8] El Karoui, N., Jeanblanc-Picqué, M. and Shreve, S. (1998). Robustness of the Black and Scholes formula. Math. Finance 8, 93-126.

[9] Föllmer, H. (1981). Calcul d'Itô sans probabilités. In Séminaire de probabilités XV (Lecture Notes Math. 850), Springer, Berlin, pp. 143-150.

[10] Föllmer, H. (1991). Probabilistic aspects of options. Rolf Nevanlinna Institute Reports B6, Helsinki.

[11] Föllmer, H. (2001). Probabilistic aspects of financial risk. In European Congress of Mathematics (Barcelona 2000; Progr. Math. 201), Vol. I, Birkhäuser, Basel, pp. 21-36.

[12] Föllmer, H. ANd Schied, A. (2004). Stochastic Finance. An Introduction in Discrete Time (de Gruyter Studies Math. 27), 2nd edn. De Gruyter, Berlin.

[13] Gushchin, A. And Mordecki, È. (2002). Bounds on option prices for semimartingale market models. Proc. Steklov Inst. Math. 2002, 73-113.

[14] Gyöngy, I. (1986). Mimicking the one-dimensional marginal distributions of processes having an Itô differential. Prob. Theory Relat. Fields 71, 501-516.

[15] HajeK, B. (1985). Mean stochastic comparison of diffusions. Z. Wahrscheinlichkeitsth. 68, 315-329.

[16] Hardy, G. H., Littlewood, J. E. And Pólya, G. (1952). Inequalities, 2nd edn. Cambridge University Press.

[17] Hobson, D. (1998). Volatility misspecification, option pricing and superreplication via coupling. Ann. Appl. Prob. 8, 193-205.

[18] Janson, S. And Tysk, J. (2003). Volatility time and properties of option prices. Ann. Appl. Prob. 13, 890-913.

[19] Janson, S. And Tysk, J. (2004). Preservation of convexity to solutions of parabolic equations. J. Differential Equat. 206, 182-226.

[20] Karatzas, I. And Shreve, S. E. (1991). Brownian Motion and Stochastic Calculus (Graduate Texts Math. 113), 2nd edn. Springer, Berlin.

[21] LyONS, T. (1995). Uncertain volatility and the risk-free synthesis of derivatives. Appl. Math. Finance 2, 117-133.

[22] Schied, A. (2005). Optimal investments for robust utility functionals in complete market models. Math. Operat. Res. 30, 750-764.

[23] Stadje, M. (2005). Convexity of option prices in a local volatility model. Diploma thesis, TU Berlin.

[24] Wright, E. M. (1954). An inequality for convex functions. Amer. Math. Monthly 61, 620-622. 